\title{
Depressive symptoms and the associations with individual, psychosocial, and structural determinants in Swedish adolescents
}

\author{
Heléne Z. Dahlqvist ${ }^{*}$, Evelina Landstedt, Katja Gillander Gådin \\ Department of Health Sciences, Mid Sweden University, Sundsvall, Sweden; \\ ${ }^{*}$ Corresponding Author: helene.zetterstrom-dahlqvist@miun.se
}

Received 27 June 2012; revised 25 July 2012; accepted 10 August 2012

\begin{abstract}
Depressive symptoms in adolescents are an increasing public health issue in Sweden and in most Western countries. Aim: To explore how individual, psychosocial, and structural determinants are associated with depressive symptoms in Swedish adolescents. Methods: A webbased questionnaire was answered by 1193 13to 16-year-old boys $(n=566)$ and girls $(n=627$ ). Stepwise logistic regressions were employed to analyse the association between depressive symptoms and various determinants at the individual level (self-efficacy), the psychosocial level (parental, peer, and teacher support, school demands, sexual harassment, and bullying) and the structural level (family affluence, having less money than friends, and parental foreign background). Results: Determinants at the individual, psychosocial, and structural levels were independently associated with high levels of depressive symptoms in both boys and girls. The full model explained a high proportion of the variance in depressive symptoms in both genders; $34.1 \%$ in boys and $36.8 \%$ in girls. The psychosocial level contributed the most to explaining the variance in depressive symptoms in boys. In girls, when harassment variables were separated from psychosocial variables, the harassment variables contributed as much to the full model as the rest of the psychosocial variables combined. Conclusions: Addressing psychosocial determinants provides the greatest benefits for preventing depressive symptoms in adolescents. Acknowledging the association between sexual harassment and depressive symptoms for girls and having less money than their friends for boys and girls are particularly important.
\end{abstract}

Keywords: Mental Health; Individual Determinants;
Psychosocial Determinants; Structural Determinants; Socioeconomic Factors; Gender; Harassment; Social Support; School; Adolescence

\section{INTRODUCTION}

Psychological distress in adolescence is a major public health issue in Sweden, as in most Western countries, with increasing rates over time [1-3]. There is evidence suggesting that psychological distress in childhood increases the risk of poor mental health in adulthood [4] which in turn has an impact on socioeconomic status including education, weeks worked per year, individual earnings, and family income. If unequally distributed in any given population, these factors in themselves have been shown to affect poor health outcomes [5]. Hence, it is crucial to learn more about what factors are associated with psychological distress in adolescence in order to be able to take appropriate measures to prevent poor mental health in adulthood. Preventing poor mental health in adolescents to enable them to have as healthy a life as possible in their teenage years has a value in its own right. In the vast literature on this matter, the term psychological distress is used to include a range of states including affective (e.g., depressive symptoms, which are the focus of the present study), cognitive, behavioural, and psychosomatic aspects (e.g., [1-3]). Several studies have pointed out that adolescent girls report higher levels of psychological distress than do boys (e.g., $[1,2,6]$ ), but little is known about the causes of these reported differences. Determinants of adolescent psychological distress can be found on individual, social, and structural levels. Determinants on an individual level are factors that affect individual resilience, such as generalized self-efficacy and global self-esteem $[7,8]$. However, previous findings on the effects of low self-esteem and low self-efficacy are inconsistent, and in studies where effects have been found, they have been small [9]. Psychosocial determinants have been proposed, such as the school environment, including 
demands in school [10] and social support [11]. Furthermore, strained peer and parental relations are associated with elevated levels of psychological distress [12]. Bullying has been investigated as a determinant of psychological distress [13,14], although sexual harassment has not been addressed to the same degree. However, examples of studies of sexual harassment show that being a target of sexual harassment is common and has an impact on well being and psychological distress in adolescents [14-16]. There is evidence of socioeconomic position as being an important structural-level determinant of psychological distress $[17,18]$. In Sweden, it has been found that parental foreign background is associated with psychological distress in girls, but not in boys [19]. The current study focuses on depressive symptoms (rather than clinical depression) as an aspect of psychological distress. Considering the relatively limited contribution of individual-level determinants to explain depressive symptoms in adolescents, studies have called for multivariate models addressing a wide range of risk factors for depression among adolescents [9]. To the best of our knowledge, depressive symptoms have not previously been explored in relation to all of the above-mentioned determinants in community-dwelling adolescents aged $13-16$ in Sweden. To gain a somewhat more comprehensive understanding of the determinants associated with depressive symptoms in this age group, we developed a model to evaluate how determinants at different levels were associated with depressive symptoms in adolescents and how different variables independently contributed to explain the likelihood of reporting depressive symptoms. The overall objective of this study was to explore how individual, psychosocial, and structural determinants are associated with depresssive symptoms in Swedish adolescent boys and girls.

\section{METHODS}

\subsection{Context}

The study utilized data from a cross-sectional study of students in a municipality in the Northern part of Sweden. The municipality is of medium size, with 59,000 inhabitants, and is characterized by a diverse socioeconomic base. There is a strong emphasis on tourism and support for small- and medium-sized enterprises. Compared to Sweden in general, the municipality has fewer inhabitants with foreign backgrounds and it is situated close to the Norwegian border, implying a relatively high proportion of immigrants from Norway.

\subsection{Participants}

All public and independent junior high schools with students in grades 7 - 9 (13 - 16 year olds) were invited to participate. Nine public junior high schools, which rep- resent all public junior high schools in the municipality and one out of the four independent schools, participated in the study. In total, this study included 1193 respondents ( $47.4 \%$ boys, $52.6 \%$ girls) giving a response rate of $80.49 \%$.

\subsection{Procedure}

An information letter was sent to the students and their parents to ensure informed consent. The identities of the respondents were confidential. The project manager kept the identities of the students in this baseline study in a register for a longitudinal follow-up. The study was approved by the Umeå Regional Ethical Review Board as being in accordance with ethical standards of research (Dnr 09-179M). The data were collected using a self-administered, web-based questionnaire that is a part of a larger study aimed at increasing knowledge about mental health in adolescents. The students were provided with a link to the questionnaire via their school e-mail addresses. In the e-mail, the students had the opportunity to decline participation and to avoid receiving a reminder. The questionnaires were completed during school hours in the presence of an adult who could answer the students' questions and ensured that they were able to complete the questionnaire individually and without disturbances. The nonrespondents differed between the schools by $10 \%-40 \%$, mainly due to a lack of engagement by the school administration in providing adequate time and space to fill in the questionnaire in different classes. Fourteen students actively declined to participate.

\subsection{Measures}

The details of the survey items used and the data reduction technique are presented in Table 1. To facilitate model interpretation, all independent variables were dichotomized or trichotomized. Multiple-item indices were computed by standardizing and averaging constituent items and were then categorized based on the distribution of responses (i.e., tertiles or empirical mean), or categories were collapsed into larger levels, such as high, moderate, and low levels of exposure. For all independent variables, the categories hypothesized to predict the lowest levels of risk for depressive symptoms were set as the reference categories.

\subsection{Statistical Analyses}

The SPSS 18 for Windows was used for all statistical analyses. Chi-squared tests were used to test differences in prevalence between genders. Logistic regression in three steps was employed to estimate odds ratios (OR) [25]. First, the individual-level determinants block was entered (Model 1). Next the psychosocial determinants block, excluding the harassment items, was entered (Model 2a), 
Table 1. Construction of analytic variables.

\begin{tabular}{|c|c|c|c|}
\hline Analytic variable & Instrument or wording of items used & Reduction/categorization & Analytic variable levels \\
\hline \multicolumn{4}{|l|}{$\begin{array}{l}\text { Individual } \\
\text { determinants }\end{array}$} \\
\hline Self-efficacy & $\begin{array}{l}\text { Generalized self-efficacy scale [20]. Likert-scale: strongly agree, } \\
\text { agree, disagree or strongly disagree. Correlates to self-esteem r: } 0.35 \text {. }\end{array}$ & $\begin{array}{l}\text { Dichotomized using the } \\
\text { empirical mean of } 29\end{array}$ & $\begin{array}{l}<29 \text { lower self-efficacy. } \\
\geq 29 \text { higher self-efficacy. }\end{array}$ \\
\hline Self-esteem & $\begin{array}{l}\text { Global self-esteem (GSE), The Rosenberg Self-Esteem Inventory } \\
\text { (RSE) [21]. Likert-scale: strongly agree, agree, disagree or strongly } \\
\text { disagree. Correlates to depressive symptoms r: } 0.49 \text {. }\end{array}$ & Trichotomized & $\begin{array}{l}\text { High GSE: } \geq 26 \\
\text { Middle GSE: } \geq 15 \\
\text { Low GSE: } \leq 14\end{array}$ \\
\hline \multicolumn{4}{|l|}{$\begin{array}{l}\text { Psychosocial } \\
\text { determinants }\end{array}$} \\
\hline Parental support & $\begin{array}{l}\text { "Do you usually talk about most things with your... mother; father?" } \\
\text { 6-point scale: always, often, sometimes, rarely, never, don't have } \\
\text { (either of the above)/never see (either of them). }\end{array}$ & Dichotomized & $\begin{array}{l}\text { Yes (always, often). } \\
\text { No (sometimes, rarely, never, } \\
\text { don't have/ never see (either } \\
\text { of them)). }\end{array}$ \\
\hline Peer support & $\begin{array}{l}\text { "Do you have as many friends you would like?"; "Do you usually talk } \\
\text { about most things with your friends?" A 5-point scale: always, often, } \\
\text { sometimes, rarely, never. Cronbach } \alpha \text { : } 0.32 \text {. }\end{array}$ & $\begin{array}{l}\text { Trichotomized using } \\
\text { tertiles }\end{array}$ & $\begin{array}{l}\text { High: }<3 \\
\text { Middle: }=4 \\
\text { Low: } \geq 5\end{array}$ \\
\hline Teacher support & $\begin{array}{l}\text { "Do your teachers give you support and help when you need it?"; "Do } \\
\text { you think your teachers would notice if you didn't get on with } \\
\text { school?"; "Do you think that your teachers treat you fairly?"; "Do } \\
\text { your teachers praise or encourage you?". 5-point scale: always, often, } \\
\text { sometimes, rarely, never. Cronbach } \alpha: 0.87 \text {. }\end{array}$ & $\begin{array}{l}\text { Trichotomized using } \\
\text { tertiles }\end{array}$ & $\begin{array}{l}\text { High: } \geq 16 \\
\text { Middle: } \geq 13 \\
\text { Low: } \leq 12\end{array}$ \\
\hline School demands & $\begin{array}{l}\text { "School work moves forward to quickly"; "What we are supposed to } \\
\text { learn is too difficult"; "The teachers give us too much homework" A } \\
\text { 5-point scale: always, often, sometimes, rarely, never [22]. }\end{array}$ & $\begin{array}{l}\text { Trichotomized using } \\
\text { tertiles }\end{array}$ & $\begin{array}{l}\text { High: } \geq 11 \\
\text { Middle: } \leq 10 \\
\text { Low: } \leq 8\end{array}$ \\
\hline $\begin{array}{l}\text { Sexual } \\
\text { harassment }\end{array}$ & $\begin{array}{l}\text { Fourteen items based on Gruber et al. [14]. To fit in a Swedish con- } \\
\text { text, one item was removed; "Listed you in a "slam book" with sexual } \\
\text { remarks about you". Frequency the last six months on a 4-point scale: } \\
\text { never, once, a few times, several times. }\end{array}$ & Trichotomized & $\begin{array}{l}\text { Never exposed. } \\
\text { Moderately: } 1 \text { behaviour once. } \\
\text { High: } \geq 2 \text { behaviours a few or } \\
\text { several times. }\end{array}$ \\
\hline Bullying & $\begin{array}{l}\text { "It happens that one or several students tease, pick fights or shut } \\
\text { somebody out. Have any of that happened to you in the last six } \\
\text { months?" A 5-point scale: Yes, almost all the time; Yes, several times; } \\
\text { Yes, a few times; Yes, once; No, never. }\end{array}$ & Dichotomized & $\begin{array}{l}\text { Never exposed. } \\
\text { Exposed: exposed almost all } \\
\text { the time, several times, a few } \\
\text { times, or once. }\end{array}$ \\
\hline \multicolumn{4}{|l|}{$\begin{array}{l}\text { Structural } \\
\text { determinants }\end{array}$} \\
\hline \multirow[t]{2}{*}{ Socioeconomy } & Family affluence scale (FAS) [23]. & $\begin{array}{l}\text { Trichotomized using } \\
\text { tertiles }\end{array}$ & \multirow{2}{*}{$\begin{array}{l}\text { High FAS: } 7 . \\
\text { Middle FAS: } 6 . \\
\text { Low FAS: } 0-5 . \\
\text { Yes (always, often). } \\
\text { No (sometimes, rarely, never). }\end{array}$} \\
\hline & $\begin{array}{l}\text { "If you consider your situation in the past six months, have you had } \\
\text { enough money to be able to do the same things as your friends?" A } \\
5 \text {-point scale: always, often, sometimes, rarely, never. }\end{array}$ & Dichotomized & \\
\hline $\begin{array}{l}\text { Parental foreign } \\
\text { background }\end{array}$ & $\begin{array}{l}\text { In what country was your mother born? } \\
\text { In what country was your father born? }\end{array}$ & Dichotomized & $\begin{array}{l}\text { No, both born in Sweden. } \\
\text { Yes: one/both parents born in } \\
\text { another country. }\end{array}$ \\
\hline $\begin{array}{l}\text { Depressive } \\
\text { symptoms }\end{array}$ & $\begin{array}{l}\text { The CES-D scale [24]. Items are formulated as a statement using a } \\
\text { 4-point scale: rarely/never, sometimes, often, always. }\end{array}$ & Dichotomized & A cut off score of $\leq 16$. \\
\hline School year & What school year are you in? & Trichotomized & $7,8,9$ \\
\hline
\end{tabular}

followed by the psychosocial determinants block, including the harassment items (Model 2b). Finally, all variables, including the structural determinants block, were included in the model (Model 3) to analyse the contribution of each variable independently while controlling for all other variables. To explore whether these variables contributed differently to the model by gender the regres- sions were run separately for boys and girls. Confidence intervals of $95 \%$ were used and the significance level was set to $p<0.05$. Analyses of correlation were performed for all variables included in the regression. Due to high correlation $(\mathrm{r}=0.49)$ between low self-esteem and high levels of depressive symptoms, self-esteem was not included in the regression analysis. 


\section{RESULTS}

\subsection{Descriptive Statistics}

As shown in Table 2, the prevalence of high levels of depressive symptoms was $23.0 \%$ among boys and almost twice as high among girls $(44.3 \%, p<0.001)$. With regard to the independent variables, significant differences between the genders were found in self-efficacy, selfesteem, parental and peer support, bullying and family affluence.

Table 2. Prevalence in each variable in boys and girls.

\begin{tabular}{|c|c|c|c|}
\hline Predictor & $\begin{array}{c}\text { Boys }\left(\mathrm{n}^{\mathrm{a}}=566\right) \\
\%(\mathrm{n})\end{array}$ & $\begin{array}{c}\text { Girls }\left(\mathrm{n}^{\mathrm{a}}=627\right) \\
\%(\mathrm{n})\end{array}$ & $p$-value \\
\hline $\begin{array}{c}\text { Outcome variable } \\
\text { Depressive symptoms }\end{array}$ & & & \multirow[b]{2}{*}{0.000} \\
\hline $\begin{array}{l}\text { No } \\
\text { Yes }\end{array}$ & $\begin{array}{l}77.0(412) \\
23.0(123)\end{array}$ & $\begin{array}{l}55.7(343) \\
44.3(273)\end{array}$ & \\
\hline $\begin{array}{l}\text { Individual determinants } \\
\text { Self-efficacy }\end{array}$ & & & \\
\hline $\begin{array}{l}\text { High } \\
\text { Low }\end{array}$ & $\begin{array}{l}59.2(298) \\
40.8(205)\end{array}$ & $\begin{array}{l}51.4(302) \\
48.6(285)\end{array}$ & 0.010 \\
\hline Self-esteem & & & \multirow[b]{2}{*}{0.000} \\
\hline $\begin{array}{l}\text { High } \\
\text { Middle } \\
\text { Low }\end{array}$ & $\begin{array}{c}38.8(205) \\
53.1(281) \\
8.1(43)\end{array}$ & $\begin{array}{l}18.6(113) \\
61.5(374) \\
19.9(121)\end{array}$ & \\
\hline $\begin{array}{c}\text { Psychosocial determinan } \\
\text { Parental support }\end{array}$ & & & \multirow[b]{2}{*}{0.013} \\
\hline $\begin{array}{l}\text { High } \\
\text { Middle } \\
\text { Low }\end{array}$ & $\begin{array}{l}41.3(187) \\
25.4(115) \\
33.3(151)\end{array}$ & $\begin{array}{l}34.5(193) \\
33.5(187) \\
32.0(179)\end{array}$ & \\
\hline Peer support & & & \\
\hline $\begin{array}{l}\text { High } \\
\text { Middle } \\
\text { Low }\end{array}$ & $\begin{array}{l}34.8(158) \\
21.1(96) \\
44.1(200)\end{array}$ & $\begin{array}{l}46.3(290) \\
17.4(109) \\
31.4(183)\end{array}$ & 0.000 \\
\hline $\begin{array}{c}\text { Teacher support } \\
\text { High } \\
\text { Middle } \\
\text { Low }\end{array}$ & $\begin{array}{l}38.6(177) \\
26.9(123) \\
34.5(158)\end{array}$ & $\begin{array}{l}32.2(183) \\
32.5(185) \\
35.3(201)\end{array}$ & 0.055 \\
\hline $\begin{array}{c}\text { School demands } \\
\text { Low } \\
\text { Middle } \\
\text { High }\end{array}$ & $\begin{array}{l}38.9(201) \\
25.9(134) \\
35.2(182)\end{array}$ & $\begin{array}{l}37.8(229) \\
31.0(188) \\
31.2(189)\end{array}$ & 0.137 \\
\hline $\begin{array}{l}\text { Sexual harassment } \\
\text { No experience } \\
\text { Moderate experience } \\
\text { High experience }\end{array}$ & $\begin{array}{c}54.9(271) \\
26.1(129) \\
19.0(94)\end{array}$ & $\begin{array}{l}48.4(278) \\
30.6(175) \\
21.0(121)\end{array}$ & 0.107 \\
\hline $\begin{array}{c}\text { Bullying } \\
\text { No } \\
\text { Yes }\end{array}$ & $\begin{array}{l}73.7(365) \\
26.3(130)\end{array}$ & $\begin{array}{l}65.5(389) \\
34.5(205)\end{array}$ & 0.002 \\
\hline $\begin{array}{c}\text { Structural determinants } \\
\text { FAS } \\
\text { High } \\
\text { Middle } \\
\text { Low }\end{array}$ & $\begin{array}{l}29.8(165) \\
28.2(156) \\
42.0(232)\end{array}$ & $\begin{array}{l}29.5(183) \\
34.4(213) \\
36.1(224)\end{array}$ & 0.048 \\
\hline $\begin{array}{c}\text { Money as friends } \\
\text { Yes } \\
\text { No }\end{array}$ & $\begin{array}{l}82.4(463) \\
17.6(99)\end{array}$ & $\begin{array}{l}80.0(497) \\
20.0(124)\end{array}$ & 0.302 \\
\hline $\begin{array}{l}\text { Parental foreign } \\
\text { background }\end{array}$ & & & \\
\hline $\begin{array}{l}\text { No } \\
\text { Yes }\end{array}$ & $\begin{array}{l}87.1(484) \\
12.9(72)\end{array}$ & $\begin{array}{l}83.8(521) \\
16.2(101)\end{array}$ & 0.111 \\
\hline
\end{tabular}

${ }^{\text {a }}$ Differences to the total $\mathrm{n}$ (1193) are due to missing cases.

\subsection{Associations between Different Levels of Determinants and Depressive Symptoms}

The results from the logistic regressions (Models 1 - 3) are presented in Table $\mathbf{3}$ for boys and Table 4 for girls.

\subsubsection{Individual Determinant}

The individual determinant of self-efficacy (Model 1) explained $10.1 \%$ and $8.0 \%$ of the variation in depressive symptoms for boys and girls respectively. In the full model (Model 3), reporting low self-efficacy remained independently associated with high levels of depressive symptoms in both genders with odds ratios increasing two and a half times in boys and nearly two times in girls.

\subsubsection{Psychosocial Determinants}

As shown in Tables 3 and 4, psychosocial determinants (Model 2a) added $11.5 \%$ to the explanation of the model in boys and $12.1 \%$ in girls, explaining thus far $21.6 \%$ of the variation in depressive symptoms in boys and $20.1 \%$ in girls. When the harassment items were added in Model $2 \mathrm{~b}$, these variables contributed to the explanation of the variance in the outcome variable $(6.5 \%$ in boys and $13.6 \%$ in girls), giving a total explanation in Model $2 \mathrm{~b}$ of $28.1 \%$ in boys and $33.7 \%$ in girls. Among boys, the psychosocial variables independently associated with high levels of depressive symptoms in the full model (Model 3) were low teacher support and bullying as both of these variables increased the odds ratios two and a half times. In comparison with boys, a greater number of psychosocial variables generated significant odds ratios in the full model in girls (Model 3); low parental support, low teacher support, high school demands and moderate and high experience of sexual harassment and bullying. The strongest association in girls was found for experiences of high levels of sexual harassment, which increased the odds ratios five and a half times. Experiences of low parental and teacher support, high school demands and moderate experience of sexual harassment and bullying increased the odds ratios about two times. The odds ratios for peer support were not significant in the full model for boys or girls. Furthermore, for boys, parental support, school demands and sexual harassment did not show statistically significant odds ratios in the full model.

\subsubsection{Structural Determinants}

The last block, structural determinants, added $6.0 \%$ and $3.1 \%$ to the explanation of the variation in depressive symptoms to the full model for boys and girls, respectively (see Tables 3 and 4). The full model (Model 3) explained $34.1 \%$ of the variation in depressive symptoms in boys and $36.8 \%$ in girls. For boys, having less money than their friends increased the likelihood of reporting depressive symptoms by about three and a half times and 
Table 3. Odds ratios (ORs) for determinants potentially associated with high levels of depressive symptoms in boys.

\begin{tabular}{|c|c|c|c|c|c|c|}
\hline $\begin{array}{l}\text { Predictors/ } \\
\text { determinants }\end{array}$ & $\begin{array}{l}\text { Boys with } \\
\text { depressive } \\
\text { symptoms } \\
\geq 16 \% \text { (n) }\end{array}$ & Crude $\mathrm{OR}^{\mathrm{a}}$ & $\begin{array}{l}\text { Model } 1 \\
\text { Individual } \\
\text { determinant }^{\mathrm{a}}\end{array}$ & $\begin{array}{c}\text { Model 2a } \\
\text { Individual \& } \\
\text { psychosocial determinants }^{\mathrm{a}}\end{array}$ & $\begin{array}{c}\text { Model 2b } \\
\text { Individual \& psychosocial } \\
\text { determinants including } \\
\text { harassment }^{\text {ac }}\end{array}$ & $\begin{array}{c}\text { Model } 3 \\
\text { Individual, psychosocial, } \\
\text { harassment \& structural } \\
\text { determinants }^{\mathrm{a}} \\
\end{array}$ \\
\hline & & OR $(95 \% \mathrm{CI})$ & OR $(95 \% \mathrm{CI})$ & OR $(95 \% \mathrm{CI})$ & OR $(95 \% \mathrm{CI})$ & OR $(95 \% \mathrm{CI})$ \\
\hline $\begin{array}{l}\text { Individual det. }^{\mathrm{d}} \\
\text { Self-efficacy }\end{array}$ & & & & & & \\
\hline $\begin{array}{l}\text { High } \\
\text { Low }\end{array}$ & $\begin{array}{l}13.4(39) \\
35.5(72)\end{array}$ & $\begin{array}{c}1.00 \\
3.61^{* * *} \\
(2.32-5.64)\end{array}$ & $\begin{array}{c}1.00 \\
3.61^{* * *} \\
(2.32-5.64)\end{array}$ & $\begin{array}{c}1.00 \\
2.72^{* * *} \\
(1.55-4.76)\end{array}$ & $\begin{array}{c}1.00 \\
2.87^{* *} \\
(1.58-5.20)\end{array}$ & $\begin{array}{c}1.00 \\
2.48^{*}(1.32-4.65)\end{array}$ \\
\hline $\begin{array}{c}\mathrm{R}^{2} \text { change } \\
\text { Psychosocial det. } \\
\text { Parental support }\end{array}$ & & - & 10.1 & & & \\
\hline High & $12.5(23)$ & 1.00 & & 1.00 & 1.00 & 1.00 \\
\hline Middle & $21.4(24)$ & $1.91^{*}(1.02-3.57)$ & & $1.56(0.74-3.26)$ & $1.45(0.66-3.17)$ & $1.17(0.52-2.65)$ \\
\hline Low & $28.1(41)$ & $\begin{array}{c}2.81^{* * *} \\
(1.59-4.98)\end{array}$ & & $2.20^{*}(1.10-4.41)$ & $2.05(0.98-4.27)$ & $1.34(0.61-2.95)$ \\
\hline $\begin{array}{l}\text { Peer support } \\
\text { High }\end{array}$ & $13.0(20)$ & 1.00 & & 1.00 & 1.00 & 1.00 \\
\hline Middle & $17.7(17)$ & $1.43(0.71-2.90)$ & & $1.32(0.57-3.06)$ & $1.14(0.48-2.76)$ & $1.32(0.53-3.30)$ \\
\hline Low & $27.6(54)$ & $2.54^{* *}(1.44-4.48)$ & & $2.23^{*}(1.12-4.43)$ & $2.01(0.98-4.12)$ & $1.84(0.86-3.92)$ \\
\hline $\begin{array}{c}\text { Teacher support } \\
\text { High }\end{array}$ & $13.1(23)$ & 1.00 & & 1.00 & 1.00 & 1.00 \\
\hline Middle & $14.0(17)$ & $1.10(0.56-2.15)$ & & $0.94(0.44-3.03)$ & $0.93(0.42-2.09)$ & $0.81(0.34-1.91)$ \\
\hline Low & $35.9(55)$ & $\begin{array}{c}3.95^{* * *} \\
(2.26-6.90)\end{array}$ & & $\begin{array}{c}2.53^{* *} \\
(1.29-4.96)\end{array}$ & $2.24^{*}(1.10-4.55)$ & $2.53^{*}(1.18-5.43)$ \\
\hline $\begin{array}{l}\text { School demands } \\
\text { Low }\end{array}$ & $14.9(29)$ & 1.00 & & 1.00 & 1.00 & 1.00 \\
\hline Middle & $25.0(33)$ & $1.91^{*}(1.09-3.34)$ & & $1.31(0.65-2.66)$ & $1.22(0.58-2.59)$ & $1.18(0.54-2.58)$ \\
\hline High & $28.4(50)$ & $\begin{array}{c}2.30^{* *} \\
(1.38-3.86)\end{array}$ & & $1.29(0.66-2.52)$ & $1.33(0.66-2.70)$ & $0.95(0.45-2.04)$ \\
\hline $\begin{array}{c}\mathrm{R}^{2} \text { change }{ }^{\mathrm{b}} \\
\text { Sexual harassment }\end{array}$ & & - & & 11.5 & & \\
\hline No & $14.0(37)$ & 1.00 & & & 1.00 & 1.00 \\
\hline Moderate & $22.8(29)$ & $1.82^{*}(1.06-3.14)$ & & & $1.49(0.76-2.90)$ & $1.38(0.68-2.82)$ \\
\hline High & $45.7(42)$ & $\begin{array}{c}5.22^{* * *} \\
(3.04-8.96)\end{array}$ & & & $1.98(0.92-4.23)$ & $1.82(0.81-4.09)$ \\
\hline Bullying & & & & & & \\
\hline $\begin{array}{l}\text { No } \\
\text { Yes }\end{array}$ & $\begin{array}{l}15.3(55) \\
40.8(51)\end{array}$ & $\begin{array}{c}1.00 \\
3.79^{* * *} \\
(2.40-5.99)\end{array}$ & & & $\begin{array}{c}1.00 \\
2.77^{* *}(1.48-5.19)\end{array}$ & $\begin{array}{c}1.00 \\
2.58^{* *} \\
(1.32-5.07)\end{array}$ \\
\hline $\begin{array}{l}\mathrm{R}^{2} \text { change }^{\mathrm{b}} \\
\text { Structural det. } \\
\text { FAS } \\
\text { High }\end{array}$ & $18.2(28)$ & - & & & 6.5 & 1.00 \\
\hline Middle & $21.9(33)$ & $1.26(0.72-2.21)$ & & & & $1.14(0.51-2.59)$ \\
\hline Low & $26.5(58)$ & $1.62(0.97-2.69)$ & & & & $1.62(0.74-3.51)$ \\
\hline $\begin{array}{c}\text { Money as friends } \\
\text { Yes }\end{array}$ & $17.5(77)$ & 1.00 & & & & 1.00 \\
\hline No & $36.4(44)$ & $\begin{array}{c}4.37^{* * *} \\
(2.71-7.05)\end{array}$ & & & & $\begin{array}{c}3.73^{* * *} \\
(1.80-7.74)\end{array}$ \\
\hline $\begin{array}{l}\text { Parental foreign } \\
\text { background }\end{array}$ & & & & & & \\
\hline Yes & $\begin{array}{l}19.1(88) \\
47.8(32)\end{array}$ & $\begin{array}{c}1.00 \\
3.88^{* * *} \\
(2.28-6.60)\end{array}$ & & & & $\begin{array}{c}1.00 \\
2.72^{*}(1.17-6.31)\end{array}$ \\
\hline $\begin{array}{l}\mathrm{R}^{2} \text { change } \mathrm{e}^{\mathrm{b}} \\
\text { Total } \mathrm{R}^{2^{\mathrm{b}}}\end{array}$ & & $\begin{array}{l}- \\
-\end{array}$ & 10.1 & 21.6 & 28.1 & $\begin{array}{c}6.0 \\
34.1 \\
\end{array}$ \\
\hline
\end{tabular}

${ }^{\mathrm{a}}$ Controlled for school year; ${ }^{*} p<0.05 ;{ }^{* *} p<0.01 ;{ }^{* * *} p<0.001$; ${ }^{\mathrm{b}}$ Nagelkerke $\mathrm{R}^{2}$; ${ }^{\mathrm{c}}$ Sexual harassment and bullying; ${ }^{\mathrm{d}}$ det.: determinants. 
Table 4. Logistic regression to predict reporting of high levels of depressive symptoms in girls.

\begin{tabular}{|c|c|c|c|c|c|c|}
\hline $\begin{array}{l}\text { Predictors/ } \\
\text { determinants }\end{array}$ & $\begin{array}{l}\text { Girls with } \\
\text { depressive } \\
\text { symptoms } \\
\geq 16 \% \text { (n) }\end{array}$ & Crude $\mathrm{OR}^{\mathrm{a}}$ & $\begin{array}{l}\text { Model 1 } \\
\text { Individual } \\
\text { determinant }^{\mathrm{a}}\end{array}$ & $\begin{array}{c}\text { Model 2a } \\
\text { Individual \& } \\
\text { psychosocial determinants }^{\mathrm{a}}\end{array}$ & $\begin{array}{c}\text { Model } 2 \mathrm{~b} \\
\text { Individual \& psychosocial } \\
\text { determinants including } \\
\text { harassment }^{\mathrm{ac}}\end{array}$ & $\begin{array}{c}\text { Model } 3 \\
\text { Individual, psychosocial, } \\
\text { harassment \& structural } \\
\text { determinants }^{\mathrm{a}}\end{array}$ \\
\hline & & OR $(95 \% \mathrm{CI})$ & OR $(95 \% \mathrm{CI})$ & OR $(95 \% \mathrm{CI})$ & OR $(95 \% \mathrm{CI})$ & OR $(95 \% \mathrm{CI})$ \\
\hline \multicolumn{7}{|l|}{ Individual det. ${ }^{\mathrm{d}}$} \\
\hline Self-efficacy & & & & & & \\
\hline High & $33.7(101)$ & 1.00 & 1.00 & 1.00 & 1.00 & 1.00 \\
\hline Low & $56.0(159)$ & $\begin{array}{c}2.57^{* * *} \\
(1.83-3.60)\end{array}$ & $\begin{array}{c}2.57^{* * *} \\
(1.83-3.60)\end{array}$ & $\begin{array}{c}2.04^{* * *} \\
(1.39-3.01)\end{array}$ & $\begin{array}{c}2.05^{* *} \\
(1.34-3.15)\end{array}$ & $\begin{array}{c}1.91^{* *} \\
(1.23-2.96)\end{array}$ \\
\hline $\mathrm{R}^{2}$ change ${ }^{\mathrm{b}}$ & & - & 8.0 & & & \\
\hline \multicolumn{7}{|l|}{ Psychosocial det. ${ }^{\mathrm{d}}$} \\
\hline $\begin{array}{c}\text { Parental support } \\
\text { High }\end{array}$ & $29.2(56)$ & 1.00 & & 1.00 & 1.00 & 1.00 \\
\hline Middle & $43.2(80)$ & $1.85^{* *}(1.21-2.84)$ & & $1.49(0.94-2.36)$ & $1.45(0.88-2.39)$ & $1.43(0.86-2.39)$ \\
\hline Low & $60.8(107)$ & $\begin{array}{c}3.80^{* * *} \\
(2.45-5.87)\end{array}$ & & $\begin{array}{c}2.72^{* * *} \\
(1.69-4.38)\end{array}$ & $1.87^{*}(1.10-3.19)$ & $1.81^{*}(1.04-3.15)$ \\
\hline \multicolumn{7}{|l|}{ Peer support } \\
\hline High & $41.8(120)$ & 1.00 & & 1.00 & 1.00 & 1.00 \\
\hline Middle & $43.1(47)$ & $1.07(0.69-1.68)$ & & $0.94(0.56-1.57)$ & $1.25(0.70-2.22)$ & $1.24(0.69-2.23)$ \\
\hline Low & $49.7(90)$ & $1.39(0.96-2.02)$ & & $1.02(0.66-1.57)$ & $1.16(0.72-1.88)$ & $1.06(0.64-1.75)$ \\
\hline \multicolumn{7}{|l|}{ Teacher support } \\
\hline High & $32.2(59)$ & 1.00 & & 1.00 & 1.00 & 1.00 \\
\hline Middle & $41.8(77)$ & $1.55^{*}(1.01-2.39)$ & & $1.20(0.74-1.93)$ & $1.27(0.75-2.15)$ & $1.28(0.74-2.21)$ \\
\hline Low & $56.3(112)$ & $\begin{array}{c}2.87^{* * *} \\
(1.88-4.38)\end{array}$ & & $1.92^{* *}(1.18-3.12)$ & $2.04^{*}(1.19-3.49)$ & $2.02^{*}(1.16-3.51)$ \\
\hline \multicolumn{7}{|l|}{ School demands } \\
\hline Low & $32.6(74)$ & 1.00 & & 1.00 & 1.00 & 1.00 \\
\hline Middle & $40.6(76)$ & $1.41(0.94-2.16)$ & & $1.06(0.66-1.68)$ & $0.91(0.55-1.51)$ & $0.96(0.57-1.62)$ \\
\hline High & $61.2(112)$ & $\begin{array}{c}3.23^{* * *} \\
(2.14-4.86)\end{array}$ & & $2.19^{* *}(1.37-3.50)$ & $1.95^{*}(1.16-3.28)$ & $1.97^{*}(1.15-3.39)$ \\
\hline $\mathrm{R}^{2}$ change $\mathrm{e}^{\mathrm{b}}$ & & - & & 12.1 & & \\
\hline \multicolumn{7}{|l|}{ Sexual harassment } \\
\hline No & $27.4(75)$ & 1.00 & & & 1.00 & 1.00 \\
\hline Moderate & $48.0(83)$ & $\begin{array}{c}2.48^{* * *} \\
(1.66-3.71)\end{array}$ & & & $1.93^{* *}(1.21-3.10)$ & $1.77^{*}(1.09-2.87)$ \\
\hline High & $71.4(85)$ & $\begin{array}{c}6.95^{* * *} \\
(4.28-11.27)\end{array}$ & & & $\begin{array}{c}5.45^{* * *} \\
(3.03-9.76)\end{array}$ & $\begin{array}{c}5.55^{* * *} \\
(3.05-10.11)\end{array}$ \\
\hline \multicolumn{7}{|l|}{ Bullying } \\
\hline No & 35.4 (136) & 1.00 & & & 1.00 & 1.00 \\
\hline Yes & $60.4(122)$ & $\begin{array}{c}2.87^{* * *} \\
(2.01-4.09)\end{array}$ & & & $\begin{array}{c}2.54^{* * *} \\
(1.63-3.96)\end{array}$ & $\begin{array}{c}2.43^{* * *} \\
(1.54-3.84)\end{array}$ \\
\hline $\mathrm{R}^{2}$ change $\mathrm{e}^{\mathrm{b}}$ & & - & & & 13.6 & \\
\hline \multicolumn{7}{|l|}{ Structural det. ${ }^{\mathrm{d}}$} \\
\hline $\begin{array}{l}\text { FAS } \\
\text { High }\end{array}$ & $43.6(79)$ & 1.00 & & & & 1.00 \\
\hline Middle & $41.2(87)$ & $0.92(0.61-1.37)$ & & & & $0.82(0.48-1.41)$ \\
\hline Low & 44.4 (106) & $1.20(0.80-1.78)$ & & & & $1.10(0.64-1.90)$ \\
\hline \multicolumn{7}{|l|}{ Money as friends } \\
\hline Yes & 38.3 (187) & 1.00 & & & & 1.00 \\
\hline No & $68.5(85)$ & $\begin{array}{c}3.55^{* * *} \\
(2.33-5.42)\end{array}$ & & & & $2.19^{* *}(1.25-3.84)$ \\
\hline $\begin{array}{l}\text { Parental foreign } \\
\text { background } \\
\text { No }\end{array}$ & $43.2(221)$ & 1.00 & & & & 1.00 \\
\hline Yes & $51.5(52)$ & $1.42(0.93-2.19)$ & & & & $1.47(0.80-2.71)$ \\
\hline $\mathrm{R}^{2}$ change ${ }^{\mathrm{b}}$ & & - & & & & 3.1 \\
\hline Total $\mathrm{R}^{2^{\mathrm{b}}}$ & & - & 8.0 & 20.1 & 33.7 & 36.8 \\
\hline
\end{tabular}

${ }^{\mathrm{a}}$ Controlled for school year; ${ }^{*} p<0.05 ;{ }^{* *} p<0.01 ;{ }^{* * *} p<0.001$; ${ }^{\mathrm{b}}$ Nagelkerke $\mathrm{R}^{2}$; ${ }^{\mathrm{c}}$ Sexual harassment and bullying; ${ }^{\mathrm{d}}$ det.: determinants. 
having parents with a foreign background increased the odds ratios two and a half times. For girls, only having less money than their friends was independently associated with depressive symptoms, increasing the likelihood of reporting depressive symptoms about two times. FAS was not significantly associated with depressive symptoms neither in boys nor in girls.

\section{DISCUSSION}

Determinants at individual, psychosocial and structural levels were independently associated with high levels of depressive symptoms in both boys and girls. The results show that the full model, with all levels included, explained a high proportion of the variance in depressive symptoms in both genders. The psychosocial level, with harassment variables excluded, contributed the most to explaining the variance of depressive symptoms in boys. However, this block also contained the greatest number of predictors which could explain these results. In contrast, for girls, the harassment variables contributed the most to the full model. The high correlation of low self-esteem with depressive symptoms and the high prevalence of depressive symptoms among the respondents with low self-esteem suggest self-esteem to be an important feature of depressive symptoms. This finding supports previous research [26], although there are inconsistencies regarding whether low self-esteem should be considered a predictor or a consequence of depressive symptoms [9]. Our results merely suggest a strong coexistence of low self-esteem with depressive symptoms in both genders. Low self-efficacy was independently associated with depressive symptoms in both boys and girls, which also is consistent with previous findings [8]. Considering that only one predictor, generalized self-efficacy, was included on the individual level, its relatively high contribution to explaining the variation in depressive symptoms supports the inclusion of this variable as a possible individual-level determinant. The relationship between high levels of depressive symptoms and low teacher support and bullying in both boys and girls is supported by previous research [14,27]. For girls, high demands in school and sexual harassment were also significantly associated with depressive symptoms. Previous research showed that high school demands may have a detrimental impact on health, but this impact can be prevented by a strong school-related sense of coherence and high levels of control [10]. However, this relationship was not examined in the current study. Both moderate and high levels of sexual harassment increased the likelihood of reporting depressive symptoms in girls. This finding is consistent with previous studies concluding sexual harassment to be a possible explanation for psychological distress $[15,16]$. However, the current study showed that being the target of only one type of sexual harassment on one single occasion was associated with depressive symp- toms in girls. This result deviates from the definition of sexual harassment used by Gruber and Fineran [14], who define being a victim of sexual harassment as exposure to at least three different types of harassment at least a few times. Our findings suggest that even low levels of experience with sexual harassment are associated with depressive symptoms. This result seems plausible in light of research showing that even witnessing sexual harassment is associated with negative mental health outcomes [16]. Contrary to previous findings [12], the relationship between peer support and depressive symptoms was not evident in boys or girls when all other factors were held constant. Surprisingly, this variable was not significant among girls in the crude model either, which is an unexpected finding. This finding may be due to differences in variable construction between studies, and should be interpreted with caution and be subjected to further investtigation. Interestingly, with regard to socioeconomic factors, the measure of relative affluence-having as much money as one's friends to be able to do the same things they do-seems to be the strongest structural predictor for depressive symptoms for both boys and girls. As shown in previous research, another relative affluence marker (pocket money), might be the socioeconomic marker that influences adolescents' ability to gain social advantages by giving individuals the ability to actively create everyday life opportunities and enhance social participation [28,29]. The inability to do the same things as one's friends because of a lack of money might lead to social exclusion and diminished possibilities to create a sense of belonging in terms of various social markers, which, as Marmot [28] argues, has a profound effect on general health. Therefore, the relative concept of having as much money as one's friends may be an important determinant of depressive symptoms in adolescence. The FAS did not contribute to the regression model for predicting reports of depressive symptoms. Other Swedish studies have also failed to show significant contributions of the FAS to subjective health complaints or multiple health complaints in multivariate analyses [30]. These findings are probably due to the relatively equal income distribution in Sweden [19]. Contrary to a previous study in Sweden [19], girls with parental foreign background did not show a significant association with depressive symptoms in the current study; however, for boys, this association was strong. It is difficult to interpret these findings, especially because the demographical characteristics of the sample in this study are not comparable to Sweden in general. Moreover, several respondents reported having parents from Norway, a Scandinavian country that is culturally similar to Sweden.

\section{LIMITATIONS}

The current study has a relatively high response rate and of the non-respondents only 14 actively declined to 
participate in the survey. However, it has been shown in previous school based surveys that mental health problems might be higher in non-attendees [31]. Hence, the prevalence reported here should be considered as a "minimum prevalence". Given the cross sectional design, conclusions on directions of causality are not possible. However, longitudinal studies of self-efficacy [8], low teacher support [11], and bullying [32] have showed an increased risk for depressive symptoms. Because the data were self-reported, the accuracy of the results depends solely on respondents' subjective assessments. Most measures used are well validated with satisfactory psychometric properties (e.g., $[14,21,24,30,33])$. To ensure reliable analyses, FAS was not coded according to the HBSC standard [30] because doing so would place only 13 boys and 15 girls in the low-FAS group. The study was predominantly based on white adolescents in a medium-sized municipality in the northern part of Sweden, which limits the possibility of generalizing the results to adolescents in general. Nevertheless, it would be reasonable to argue that the results can be generalized to junior high schools outside the major metropolitan areas in Sweden.

\section{CONCLUSION AND IMPLICATIONS FOR FURTHER RESEARCH AND PRACTICE}

The present study shows that psychosocial factors contribute the most to explaining variations in depressive symptoms among both boys and girls. For girls in particular, the association with sexual harassment was very strong, and for both boys and girls, bullying had a strong association with depressive symptoms. Hence, future longitudinal studies should consider the impact of sexual harassment and bullying to understand the development of depressive symptoms in adolescents. For public health practitioners, targeting the psychosocial determinants by working towards a health-promoting psychosocial environment in school may provide the greatest benefits in terms of depressive symptoms in adolescence. Further research on the implementation of health promotion programs in school should be conducted. Furthermore, relative affluence is an important structural issue to address in health promotion practice in adolescence, while self-efficacy seems to be an important individual-level factor. Future studies may also consider a gender perspective to enable a greater understanding of the differences between the genders regarding the impact of determinants of different levels.

\section{ACKNOWLEDGEMENTS}

The study was funded by the Swedish National Institute of Public Health. We would like to extend our gratitude to Professor Emeritus Anders Knutsson for valuable comments on the early draft of this paper and to all participating schools and students.

\section{REFERENCES}

[1] Petersen, S., Bergström, E., Cederblad, M., Ivarsson, A. Köhler, L., Rydell, A.-M., et al. (2010) Barns och ungdomars psykiska hälsa i Sverige. En systematisk litteraturöversikt med tonvikt på förändringar över tid. Stockholm: Kungliga Vetenskapsakademien.

[2] Sweeting, H., Young R. and West, P. (2009) GHQ increases among Scottish 15 year olds 1987-2006. Social Psychiatry and Psychiatric Epidemiology, 44, 579-586. doi:10.1007/s00127-008-0462-6

[3] Hagquist, C. (2010) Discrepant trends in mental health complaints among younger and older adolescents in Sweden: An analysis of WHO data 1985-2005. Journal of Adolescent Health, 46, 258-264. doi:10.1016/j.jadohealth.2009.07.003

[4] Delaney, L., Smith, J.P. (2012) Childhood health: Trends and consequences over the life course. The Future of Children, 22, 43-63. doi:10.1353/foc.2012.0003

[5] Wilkinson, R. (2005) The impact of inequality: How to make sick societies healthier. The New Press, New York.

[6] Sawyer, M.G., Pfeiffer, S. and Spence, S.H. (2009) Life events, coping and depressive symptoms among young adolescents: A one-year prospective study. Journal of $A f$ fective Disorders, 117, 48-54. doi:10.1016/j.jad.2008.12.013

[7] Schwarzer, R. and Jerusalem, M. (1995) Generalized selfefficacy scale. In: Weinman, J. Wright, S. and Johnston, M., Eds., Measures in Health Psychology: A User's Portfolio. Causal and Control Beliefs. NFER-NELSON, Windsor, 35-37.

[8] Caprara, G.V., Gerbino, M., Paciello, M., Di Giunta, L. and Pastorelli, C. (2010) Counteracting depression and delinquency in late adolescence: The role of regulatory emotional and interpersonal self-efficacy beliefs. European Psychologist, 15, 34-48. doi:10.1027/1016-9040/a000004

[9] Bohon, C., Stice, E., Burton, E., Fudell, M. and NolenHoeksema, S. (2008) A prospective test of cognitive vulnerability models of depression with adolescent girls. Behavior Therapy, 39, 79-90. doi:10.1016/j.beth.2007.05.003

[10] Modin, B., Östberg, V., Toivanen, S. and Sundell, K. (2010) Psychosocial working conditions, school sense of coherence and subjective health complaints. A multilevel analysis of ninth grade students in the Stockholm area. Journal of Adolescence, 34, 129-139. doi:10.1016/j.adolescence.2010.01.004

[11] Undheim, A. and Sund, A. (2005) School factors and the emergence of depressive symptoms among Norweigan adolescents. European Child and Adolescent Psychiatry, 14, 446-453. doi:10.1007/s00787-005-0496-1

[12] Brolin Låftman, S. and Östberg, V. (2006) The pros and cons of social relations: An analysis of adolescents' health complaints. Social Science \& Medicine, 63, 611-623. doi:10.1016/j.socscimed.2006.02.005 
http://EconPapers.repec.org/RePEc:eee:socmed:v:63:y:20 06:i:3:p:611-623

[13] Meland, E., Rydning, J.H., Lobben, S., Breidablik, H.J. and Ekeland, T.J. (2010) Emotional, self-conceptual, and relational characteristics of bullies and the bullied. Scandinavian Journal of Public Health, 38, 359-367. doi: $10.1177 / 1403494810364563$

[14] Gruber, J.E. and Fineran, S. (2007) The impact of bullying and sexual harassment on middle and high school girls. Violence Against Women, 13, 627-643. doi: $10.1177 / 1077801207301557$

[15] Gillander Gådin K. and Hammarstrom, A. (2005) A possible contributor to the higher degree of girls reporting psychological symptoms compared with boys in grade nine? European Journal of Public Health, 15, 380-385. doi:10.1093/eurpub/cki097

[16] Lichty, L.F. and Campbell, R. (2011) Targets and witnesses: Middle school students sexual harassment experiences. The Journal of Early Adolescence, 32, 414-430.

[17] Östberg, V., Alfven, G. and Hjernm, A. (2006) Living conditions and psychosomatic complaints in Swedish schoolchildren. Acta Paediatrica, 95, 929-934. doi:10.1080/08035250600636545

[18] Ravens-Sieberer, U., Erhart, M., Gosch, A. and Wille, N. (2008) Mental health of children and adolescents in 12 European countries-Results from the European KIDSCREEN study. Clinical Psychology \& Psychotherapy, 15, 154-163. doi:10.1002/cpp.574

[19] Carlerby, H., Viitasara, E., Knutsson, A. and Gillander Gådin, K. (2011) Subjective health complaints among boys and girls in the Swedish HBSC study: Focussing on parental foreign background. International Journal of Public Health, 56, 457-464. doi:10.1007/s00038-011-0246-8

[20] Scholz, U., Gutiérres-Doña, B.G., Sud, S. and Schwarzer, R. (2002) Is general self-efficacy a universal construct? Psychometric findings from 25 countries. European Journal of Psychological Assessment, 18, 242-251. doi:10.1027//1015-5759.18.3.242

[21] Rosenberg, M. (1989) Society and the adolescent selfimage. Revised Edition, Wesleyan University Press, Middletown.

[22] Gillander Gådin K. and Hammarstrom, A. (2000) School related health-A cross-sectional study among young boys and girls. International Journal of Health Services, 30, 797-820. doi:10.2190/K3EN-EAY9-GDTD-002Q
[23] Richter, M., Vereecken, C.A., Boyce, W., Maes, L., Gabhainn, S.N. and Currie, C. (2009) Parental occupation, family affluence and adolescent health behaviour in 28 countries. International Journal of Public Health, 54, 203212.ddoi:10.1007/s00038-009-8018-4

[24] Radloff, S.L. (1991) The use of the center for epidemicologic studies depression scale in adolescents and young adults. Journal of Youth and Adolescence, 20, 149-166. doi:10.1007/BF01537606

[25] Tabachnick, B.G. and Fidell, L.S. (2006) Using multivariate statistics. 5th Edition, Allyn and Bacon, Boston.

[26] Kim, Y.H. (2003) Correlation of mental health problems with psychological constructs in adolescence: Final results from a 2-year study. International Journal of Nursing Studies, 40, 115-124. doi:10.1016/S0020-7489(02)00037-8

[27] Ivarsson, T., Broberg, A., Arvidsson, T. and Gillberg, C. (2005) Bullying in adolescence: Psychiatric problems in victims and bullies as measured by the Youth Self Report (YSR) and the Depression Self-Rating Scale (DSRS). Nordic Journal of Psychiatry, 59, 365-373. doi:10.1080/08039480500227816

[28] Marmot, M. (2004) The status syndrome: How social standing affects our health and longevity. Holt McDougal, New York.

[29] Galobardes, B., Lynch, J. and Smith, G.D. (2007) Measuring socioeconomic position in health research. British Medical Bulletin, 81-82, 21-37. doi:10.1093/bmb/ldm001

[30] Currie, C., Nic Gabhainn, S., Godeau, E., Roberts, C., Smith, R., Currie, D., et al. (2008) Inequalities in young people's health: HBSC international report from the 2005/2006 survey. Child and Adolescent Health Research Unit, World Health Organisation.

[31] Edgardh, K. and Ormstad, K. (2000) Prevalence and characteristics of sexual abuse in a national sample of Swedish seventeen-year-old boys and girls. Acta Paediatrica, 89, 310-319. doi:10.1111/j.1651-2227.2000.tb01333.x.

[32] Bond, L., Carlin, J., Thomas, L., Rubin, K. and Patton, G. (2001) Does bullying cause emotional problems? A prospective study of young teenagers. British Medical Journal, 323, 480-484. doi:10.1136/bmj.323.7311.480

[33] Koskinen-Hagman, M., Schweizer, R. and Jerusalem, M. (1999) Swedish version of the general self-efficacy scale. http://userpage.fu-berlin.de/ health/swedish.htm 